\title{
Antidiabetogenic effects of hydroxychloroquine on insulin sensitivity and beta cell function: a randomised trial
}

\author{
Mary Chester M. Wasko ${ }^{1}$ - Candace K. McClure ${ }^{2} \cdot$ Sheryl F. Kelsey $^{2}$. \\ Kimberly Huber $^{3}$ - Trevor Orchard ${ }^{2,4,5}$ - Frederico G. S. Toledo ${ }^{3}$
}

Received: 22 April 2015 / Accepted: 15 June 2015 / Published online: 22 July 2015

(C) Springer-Verlag Berlin Heidelberg 2015

\begin{abstract}
Aims/hypothesis Hydroxychloroquine (HCQ), an antimalarial drug with anti-inflammatory properties, is employed in rheumatic diseases. In observational studies, patients with rheumatic diseases treated with HCQ have a lower risk of developing diabetes. However, the physiological mechanisms remain unexplained. We hypothesised that HCQ may have favourable effects on insulin sensitivity and/or beta cell function.

Methods This was a randomised, double-blind, parallel-arm (placebo vs HCQ $400 \mathrm{mg} /$ day) trial at the University of Pittsburgh. Randomisation was conducted by a computer system with concealment by sealed envelopes. Treatment duration was $13 \pm 1$ weeks. Randomised participants (HCQ $n=17$; placebo $n=15)$ were non-diabetic volunteers, age $>18$,
\end{abstract}

Electronic supplementary material The online version of this article (doi:10.1007/s00125-015-3689-2) contains peer-reviewed but unedited supplementary material, which is available to authorised users.

Mary Chester M. Wasko

mcwasko@wpahs.org

Frederico G. S. Toledo

toledofs@upmc.edu

1 Division of Rheumatology, Department of Medicine, Allegheny Health Network, 4800 Friendship Avenue, North Tower, Suite 2600, Pittsburgh, PA 15224, USA

2 Department of Epidemiology, University of Pittsburgh Graduate School of Public Health, Pittsburgh, PA, USA

3 Division of Endocrinology and Metabolism, Department of Medicine, University of Pittsburgh School of Medicine, 200 Lothrop Street BST-E1140, Pittsburgh, PA 15261, USA

4 Department of Medicine, University of Pittsburgh Graduate School of Public Health and School of Medicine, Pittsburgh, PA, USA

5 Department of Pediatrics, University of Pittsburgh Graduate School of Public Health, Pittsburgh, PA, USA overweight or obese, with one or more markers of insulin resistance. All participants were included in intention-to-treat analysis. Outcomes were changes in insulin sensitivity and beta cell function measured by intravenous glucose tolerance tests and minimal model analysis.

Results There was a positive change in insulin sensitivity with HCQ but not placebo (mean \pm SEM: $+20.0 \% \pm 7.1 \%$ vs $-18.4 \%$ $\pm 7.9 \%$, respectively; $p<0.01$; difference: $38.3 \% \pm 10.6 \%$; $95 \%$ CI: $17 \%, 60 \%$ ). Improvement in beta cell function was also observed with HCQ but not placebo $(+45.4 \% \pm 12.3 \%$ vs $-19.7 \% \pm 13.6 \% ; p<0.01$; difference: $65 \% \pm 19 \%$; $95 \%$ CI: $27 \%, 103 \%)$. There were modest treatment effects on fasting plasma glucose and $\mathrm{HbA}_{1 \mathrm{c}}(p<0.05)$ but circulating markers of inflammation (IL-6, IL-1, TNF- $\alpha$, soluble intercellular adhesion molecule) were not affected in either group. In contrast, adiponectin levels increased after HCQ treatment but not after placebo $(+18.7 \%$ vs $+0.7 \%$, respectively; $p<0.001)$. Both lowand high-molecular-weight adiponectin forms accounted for the increase. There were no serious or unexpected adverse effects. Conclusions/interpretation HCQ improves both beta cell function and insulin sensitivity in non-diabetic individuals. These metabolic effects may explain why HCQ treatment is associated with a lower risk of type 2 diabetes. An additional novel observation is that HCQ improves adiponectin levels, possibly being a mediator of the favourable effects on glucose metabolism. Our findings suggest that HCQ is a drug with considerable metabolic effects that warrant further exploration in disorders of glucose metabolism.

Trial registration Clinicaltrials.gov NCT01326533

Funding This study was funded by National Institutes of Health no. 5R21DK082878, UL1-RR024153 and UL1TR000005.

Keywords Beta cell function $\cdot$ Hydroxychloroquine $\cdot$ Insulin sensitivity 


$\begin{array}{ll}\text { Abbreviations } \\ \text { AIR } & \text { Acute insulin response } \\ \text { HCQ } & \text { Hydroxychloroquine } \\ \text { HMW } & \text { High-molecular-weight } \\ \text { LMW } & \text { Low-molecular-weight } \\ \text { s-ICAM } & \text { Soluble intercellular adhesion molecule }\end{array}$

\section{Introduction}

Prevention of type 2 diabetes mellitus remains an unmet medical challenge. While type 2 diabetes can often be prevented or delayed by lifestyle modifications that incorporate weight loss and physical activity [1,2], long-term adherence is challenging for the great majority of individuals. For this reason, there is an interest in developing pharmacological options for diabetes prevention. Insulin sensitisers, such as metformin and thiazolinidiones, have been shown to prevent or delay type 2 diabetes in large randomised clinical trials [1, 3, 4], establishing proof of concept that type 2 diabetes prevention is not only possible by pharmacological means but also worth pursuing.

Hydroxychloroquine (HCQ) is an antimalarial drug with anti-inflammatory properties that is employed in the treatment of autoimmune rheumatic diseases. An observational study of over 5,000 patients with rheumatoid arthritis suggested that HCQ may have reduced the risk of incident diabetes in those patients [5]. A proportional decrease in the risk for diabetes was seen with progressive duration of HCQ use; those on HCQ for $\geq 4$ years showed a $77 \%$ reduction in risk. Other observational studies have also suggested reductions in diabetes incidence in association with HCQ treatment in patients with rheumatic diseases $[6,7]$. Findings from these reports are consistent with the possibility that HCQ prevents type 2 diabetes, at least in patients with rheumatic diseases. Because of the observational nature of those studies, causality cannot be conclusively inferred from those studies alone. However, other lines of evidence suggest that HCQ has direct antidiabetes properties. Chloroquine, the parent compound from which HCQ originates, has been shown to have an insulin-sparing effect in type 2 diabetes $[8,9]$ and improve glucose tolerance in non-insulin-dependent diabetes [10]. The impact of HCQ on hyperglycaemia in type 2 diabetes was tested in two older randomised trials, and glucose-lowering properties were found $[11,12]$. However, the mechanisms by which HCQ may affect blood glucose and reduce the risk for type 2 diabetes have not been established.

Insulin sensitivity and beta cell function are key determinants of normal glucose homeostasis and we hypothesised that either one or both of these variables may be affected by HCQ. If confirmed, further exploration of HCQ as a drug of potential value in disorders of glucose metabolism would be warranted. Importantly, HCQ would be an appealing option because it is generic, inexpensive, easy to administer and its safety profile has been known for decades.

To examine the mechanisms of HCQ's action on glucose metabolism, we conducted a randomised clinical trial of 13 weeks of treatment with HCQ vs placebo in non-diabetic adults. The trial was primarily oriented as a mechanistic study. Glucose metabolism was studied in detail by intravenous glucose tolerance tests with minimal model analysis to examine whether HCQ improves insulin sensitivity, beta cell function or both. In addition, we also tested whether the effect of HCQ is mediated by a change in systemic inflammatory activity and adiponectin levels.

\section{Methods}

Research participants All participants provided informed consent. Protocols were approved by the University of Pittsburgh's institutional review board. The research was conducted at the University of Pittsburgh's Endocrinology and Metabolism Research Center and the Clinical Translation Research Center. All participants had a screening medical history and physical examination. Inclusion criteria were age $>18$ years, BMI $>25 \mathrm{~kg} / \mathrm{m}^{2}$ (but otherwise healthy) and one or more risk factors for insulin resistance: fasting glucose $5.51-6.88 \mathrm{mmol} / 1(100-125 \mathrm{mg} / \mathrm{dl})$; fasting insulin $>48.6 \mathrm{pmol} / \mathrm{l}(>7 \mu \mathrm{U} / \mathrm{ml})$; history of gestational diabetes or a parent with type 2 diabetes. Exclusion criteria were as follows: (1) diabetes mellitus, liver or kidney disease, myopathies, autoimmune disease, cancer, glucose-6-phosphate dehydrogenase deficiency and retinal disease; (2) pregnancy; (3) systemic corticosteroid use in the 6 months leading up to the study; (4) bariatric surgery or weight instability ( $>3 \mathrm{~kg}$ change in the previous 6 weeks); (5) metformin or drugs that affect glucose metabolism; (6) haemoglobin $<90 \mathrm{~g} \%$.

Study design and assessments This was a double-blind, randomised trial comparing HCQ vs placebo in non-diabetic individuals recruited between March 2011 and November 2012. Assuming an $\alpha$ of 0.05 , an SD of 1.13 and a power of 0.80 , a sample size of 15 participants per group was powered to detect a difference in insulin sensitivity of $0.17 \times 10^{-4}$ $\mathrm{pmol}^{-1} 1 \mathrm{~min}^{-1}$. This magnitude can be considered clinically relevant. For instance, in a study of obese individuals with impaired glucose tolerance, insulin sensitivity improved by $0.12 \times 10^{-4} \mathrm{pmol}^{-1} 1 \mathrm{~min}^{-1}$ after weight loss achieved by diet and exercise [13]. Participants were randomised to receive either once-nightly placebo $(n=15)$ or HCQ $400 \mathrm{mg}$ by mouth $(n=17)$ after baseline metabolic testing. A pharmacist who was independent of the study carried out the blinded assignment and dispensing of treatment based on a randomisation table achieved by computer-generated random numbers (blocks of four) and sealed envelopes. Treatment assignment 
(1:1) was concealed from researchers and participants until completion of study procedures. The treatment duration was $13 \pm 1$ weeks. Adherence was determined by pill counting (percentage taken from the total number of doses at study completion). During the treatment phase, four participants in the HCQ group were lost to follow-up: two lost contact; one no longer wished to participate and one left the study because of abdominal discomfort (Fig. 1).

IVGTTs Participants were admitted to the University of Pittsburgh's Clinical Translational Research Center for baseline and post-treatment metabolic assessments, conducted in the morning after an overnight fast of $8-10 \mathrm{~h}$. They were instructed to abstain from alcohol and exercise in the $48 \mathrm{~h}$ preceding these assessments. Body weight, fat mass and lean mass were measured on a bioimpedance scale (Tanita TBF-300A, Tanita, Arlington Heights, IL, USA). Metabolic assessments consisted of fasting blood collection, followed by an IVGTT to measure insulin sensitivity, insulin secretion, and beta cell function by the minimal model $[14,15]$. Two peripheral i.v. access lines were established on separate arms; 20-30 min later, baseline samples were drawn. At $T=0 \mathrm{~min}$, dextrose $50 \%$ $\mathrm{wt} / \mathrm{vol}$. ( $0.3 \mathrm{~g} / \mathrm{kg}$ by i.v. bolus) was given over $30-60 \mathrm{~s}$, followed by a bolus of insulin at $T=20 \mathrm{~min}$ (Humulin-R $20 \mathrm{mU} \mathrm{kg}^{-1}[5 \mathrm{~min}]^{-1}$ ) (Eli Lilly, Indianapolis, IN, USA). Samples were drawn for glucose and insulin measurements at $T=-10,-5,0,2,3,4,5,6,8,10,12,14,16$, $19,22,23,24,25,27,30,40,50,60,70,80,90,100$,

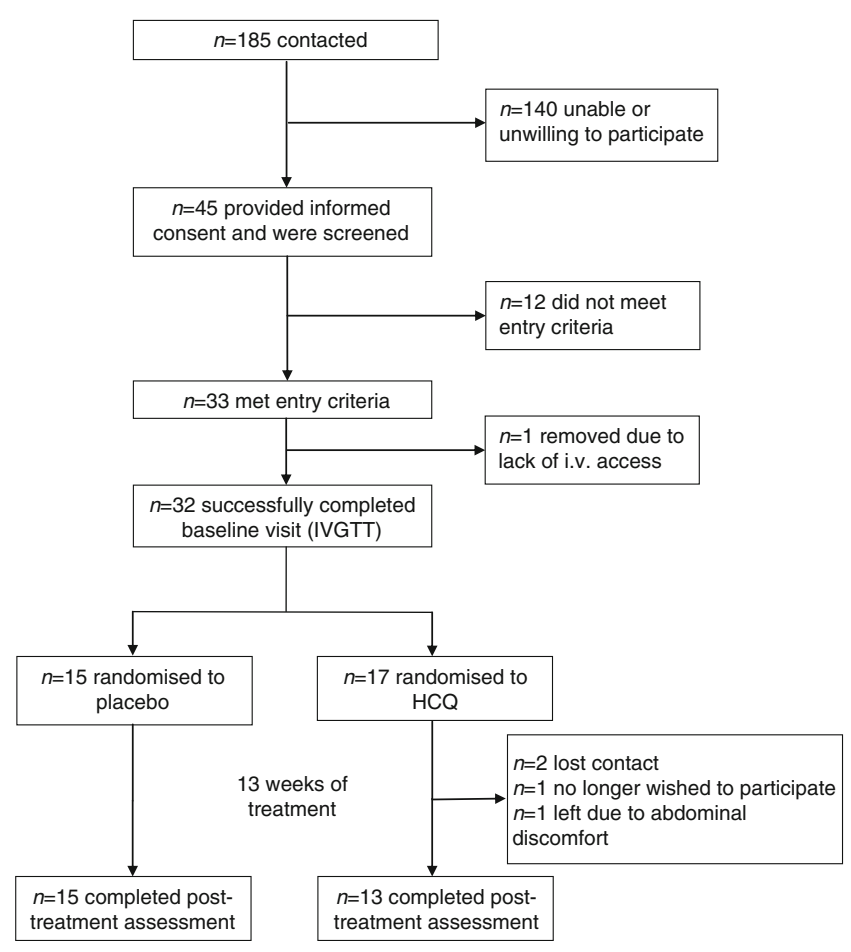

Fig. 1 Study recruitment and participation flow diagram
$120,140,160$ and $180 \mathrm{~min}$. The acute insulin response (AIR) was calculated as the integrated AUC above baseline during the first $10 \mathrm{~min}$ following exogenous dextrose administration. Minimal model analysis was employed to calculate insulin sensitivity and the disposition index, which reflects beta cell function (i.e. the ability of beta cells to regulate insulin secretion appropriate to the level of insulin resistance) $[14,15]$. The fasting molar ratio of C-peptide to insulin concentrations ( $\mathrm{pmol} / \mathrm{pmol}$ ) was calculated as a surrogate marker of insulin clearance [16]. In steady-state equilibrium conditions, this ratio estimates insulin clearance based on the assumption that insulin and C-peptide are co-secreted in equimolar proportions and that C-peptide is not subjected to first-pass hepatic metabolism [16].

Biochemical analyses Insulin was measured by a two-site chemiluminescent immunometric assay. ELISAs were employed for measurements of total and high-molecularweight (HMW) adiponectin (ALPCO Diagnostics, Salem, NH, USA). Low-molecular-weight (LMW) adiponectin was calculated as the difference between the two. ELISA kits were employed for IL-6, IL- $1 \beta$, TNF- $\alpha$ and soluble intercellular adhesion molecule-1 (s-ICAM-1) (R\&D Systems, Minneapolis, MN, USA).

Statistical analyses The Fisher's exact test, two-sample $t$ test or the Wilcoxon two-sample test was used, as appropriate, to compare characteristics between groups at baseline. The primary and secondary outcome was the change in insulin sensitivity and beta cell function, respectively. Treatment group differences were examined by ANCOVA with treatment as the fixed effect and baseline value as a covariate. Within-group changes after 13 weeks were examined by two-tailed paired $t$ test. The primary analysis was conducted on the intention-totreat population with last observation carried forward (LOCF) by imputing the baseline value as the last observation. Secondary analyses were also conducted with two distinct approaches to test different assumption scenarios. First, repeated measures analyses using linear mixed models were used to separately estimate differences in mean change in insulin sensitivity, glucose effectiveness, acute insulin sensitivity, beta cell function, glucose, and $\mathrm{HbA}_{1 \mathrm{c}}$ between the two arms. This approach used all available participant data to calculate maximum likelihood estimates for mean change in the variables listed above and accounted for repeated observations. Second, sensitivity analysis was conducted by imputing changes for participants in the HCQ group without 13 week data using the mean change observed in the placebo group. Normality of distribution was examined and confirmed by using qq and pp plots. All tests were two-sided and assessed for significance if $p<0.05$. Results are expressed as means \pm SD unless shown otherwise. 


\section{Results}

Baseline characteristics of participants At baseline, the HCQ and placebo groups had similar race distribution, sex, age, BMI and percentage of body fat (Table 1). Most participants had a parental history of type 2 diabetes (placebo $87 \%$ vs HCQ $82 \%, p=0.75$ ) and hyperinsulinaemia was similar in both groups. Fasting glucose, $\mathrm{HbA}_{1 \mathrm{c}}$, fasting NEFA and blood pressure were also similar between groups.

Effects of HCQ on glucose metabolism The number of study treatment days was similar in both groups: $94 \pm 9$ days in the placebo group vs $92 \pm 3$ days in the HCQ group $(p=0.98)$. Adherence to treatment, as determined by pill counting, was high in the placebo group (mean $99 \%$, range $80 \%-100 \%$ ) as well as in the HCQ group (mean $99 \%$, range $98 \%-100 \%$ ). Participants were weight stable as changes in weight were minimal in both groups (placebo $\Delta=+1.3 \pm 0.6 \mathrm{~kg}$ vs HCQ $\Delta=0.0 \pm 0.5 \mathrm{~kg}, p=0.11)$.

The effects of HCQ on several variables of glucose metabolism derived from the IVGTT are shown in Table 2. The insulin sensitivity index was significantly improved in the HCQ treatment group but not in the placebo group, which experienced a decline $(p<0.01)$. In secondary analyses, within-group testing confirmed that the HCQ group experienced a significant improvement in insulin sensitivity over 13 weeks $(p=0.03)$. Glucose-dependent glucose disposal $\left(\mathrm{S}_{\mathrm{G}}\right)$ (i.e. disposal that is independent of insulin) was also examined. There was no statistically significant difference between groups (Table 2). The AIR to glucose, which reflects

Table 1 Baseline characteristics of participants

\begin{tabular}{|c|c|c|c|}
\hline Characteristic & $\begin{array}{l}\text { Placebo } \\
(n=15)\end{array}$ & $\begin{array}{l}\text { HCQ } \\
(n=17)\end{array}$ & $p$ value \\
\hline Race & & & 0.780 \\
\hline White & $13(87)$ & $12(71)$ & \\
\hline Black & $1(7)$ & $5(29)$ & \\
\hline Other & $1(7)$ & 0 & \\
\hline Female sex & $9(60)$ & $15(88)$ & 0.360 \\
\hline Age (years) & $44.9 \pm 16.8$ & $50.1 \pm 14.5$ & 0.359 \\
\hline Family history of diabetes & $13(87)$ & $14(82)$ & 0.747 \\
\hline BMI $\left(\mathrm{kg} / \mathrm{m}^{2}\right)$ & $35.9 \pm 6.2$ & $34.1 \pm 7.2$ & 0.450 \\
\hline Body fat $(\%)$ & $39.5 \pm 8.7$ & $43.5 \pm 8.7$ & 0.197 \\
\hline Fasting glucose $(\mathrm{mmol} / \mathrm{l})$ & $5.2 \pm 0.5$ & $5.5 \pm 0.8$ & 0.204 \\
\hline $\mathrm{HbA}_{1 \mathrm{c}}(\%)$ & $5.65 \pm 0.41$ & $5.72 \pm 0.35$ & 0.604 \\
\hline $\mathrm{HbA}_{1 \mathrm{c}}(\mathrm{mmol} / \mathrm{mol})$ & $38 \pm 5$ & $39 \pm 4$ & 0.604 \\
\hline Fasting insulin (pmol/l) & $61.1 \pm 61.1$ & $66.7 \pm 37.5$ & 0.772 \\
\hline Fasting NEFA $(\mu \mathrm{mol} / \mathrm{l})$ & $509 \pm 150$ & $538 \pm 132$ & 0.588 \\
\hline Systolic blood pressure $(\mathrm{mmHg})$ & $127 \pm 20$ & $127 \pm 14$ & 0.978 \\
\hline Diastolic blood pressure $(\mathrm{mmHg})$ & $73 \pm 9$ & $72 \pm 13$ & 0.848 \\
\hline
\end{tabular}

Values are expressed as means \pm SD or as $n(\%)$ the absolute mass of insulin secreted, increased after 13 weeks but the difference between groups was not significant $(p=0.49)$. However, beta cell function measured by the disposition index was improved by HCQ (placebo $-19.7 \% \pm 13.6 \%$; $\mathrm{HCQ}+45.4 \pm 12.3 \% ; p=0.002$ ).

The effects of HCQ on simple markers of glucose metabolism are shown in Table 3. HCQ treatment was associated with a small but favourable effect on fasting plasma glucose concentration $(p=0.04)$ and $\mathrm{HbA}_{1 \mathrm{c}}(p=0.03)$. There was no effect on fasting insulin and free-fatty acid levels. The C-peptide/insulin molar ratio, an estimate of insulin clearance, was similar in both groups at baseline (placebo $31.4 \pm 17.1 \mathrm{vs} \mathrm{HCQ}$ $29.2 \pm 20.3, p=0.76$ ). After 13 weeks, no treatment effect was observed (placebo $-5.5 \pm 3.3$ vs HCQ $-4.8 \pm 3.6, p=0.88$ ). In agreement with this observation, IVGTT plots revealed that the profile of insulin concentration decay after exogenous insulin administration was virtually identical before and after HCQ treatment, suggestive of no change in insulin clearance (Fig. 2).

To examine the potential impact of dropouts on metabolic outcomes, two additional analyses were conducted. In mixedmodel analysis using all available data, the effect of HCQ was confirmed for insulin sensitivity $(p=0.008)$, disposition index $(p=0.009)$ and fasting glucose $(p=0.02)$. In sensitivity analyses that imputed changes for the four HCQ participants without 13 week data using the mean change observed in the placebo group, statistically significant results were confirmed for insulin sensitivity $(p=0.005)$ and the disposition index $(p=0.03)$.

Effects of HCQ on circulating biomarkers of inflammation and adiponectin Markers of inflammation were minimally affected after 13 weeks and differences between the placebo and HCQ groups were not significant (electronic supplementary material [ESM] Fig. 1). No significant changes were observed in circulating concentrations of proinflammatory cytokines (IL-1 $\beta$, IL-6 and TNF- $\alpha$ ) or s-ICAM-1 in either the placebo- or the HCQ-treated group. In sharp contrast, adiponectin was significantly increased by HCQ (Fig. 3a). Both HMW and LMW subfractions contributed to the improvement in adiponectin levels (Fig. 3b, c).

\section{Discussion}

Three observational studies showed that patients with autoimmune inflammatory arthritis treated with HCQ have a lower risk of developing diabetes [5-7]. In addition, two clinical trials reported that treatment with HCQ in high doses (600 mg/day) lowers $\mathrm{HbA}_{1 \mathrm{c}}$ in individuals with type 2 diabetes and without autoimmune conditions [11, 12]. These observations suggest that HCQ has beneficial effects on glucose metabolism. Yet, to date, the underlying 
Table 2 Changes in insulin sensitivity, glucose effectiveness, acute insulin secretion and beta cell function (disposition index)

\begin{tabular}{|c|c|c|c|c|c|}
\hline Variable & $\begin{array}{l}\text { Placebo } \\
(n=15)\end{array}$ & $\begin{array}{l}\mathrm{HCQ} \\
\left(n=17^{\mathrm{a}}\right)\end{array}$ & $\begin{array}{l}p \text { value } \\
\text { (HCQ vs } \\
\text { placebo) }\end{array}$ & $\begin{array}{l}\text { Difference } \\
\text { (HCQ vs } \\
\text { placebo) }\end{array}$ & $95 \% \mathrm{CI}$ \\
\hline \multicolumn{6}{|l|}{$\mathrm{S}_{\mathrm{I}}\left(10^{-4} \mathrm{pmol}^{-1} 1 \mathrm{~min}^{-1}\right)$} \\
\hline Baseline & $0.58 \pm 0.42$ & $0.52 \pm 0.27$ & 0.60 & & \\
\hline Change from baseline & $-0.11 \pm 0.04^{*}$ & $0.08 \pm 0.03^{*}$ & 0.001 & $0.19 \pm 0.04$ & $(0.09,0.29)$ \\
\hline$\%$ Change from baseline & $-18.4 \pm 7.9^{*}$ & $20.0 \pm 7.1^{*}$ & 0.001 & $38.3 \pm 10.6$ & $(16.6,60.1)$ \\
\hline \multicolumn{6}{|l|}{$\mathrm{S}_{\mathrm{G}}\left(\min ^{-1}\right)$} \\
\hline Baseline & $0.015 \pm 0.006$ & $0.017 \pm 0.006$ & 0.58 & & \\
\hline Change from baseline & $0.000 \pm 0.001$ & $0.004 \pm 0.001$ & 0.06 & $0.004 \pm 0.002$ & $(0.000,0.008)$ \\
\hline$\%$ Change from baseline & $29.5 \pm 40.0$ & $81.1 \pm 37.1$ & 0.36 & $51.6 \pm 55.5$ & $(-62.1,165)$ \\
\hline \multicolumn{6}{|l|}{$\operatorname{AIR}(\mathrm{pmol} / 1 \times 10 \mathrm{~min})$} \\
\hline Baseline & $2,938 \pm 2,347$ & $3,632 \pm 3,945$ & 0.56 & & \\
\hline Change from baseline & $382 \pm 236$ & $83 \pm 215$ & 0.35 & $-299 \pm 319$ & $(-361,958)$ \\
\hline$\%$ Change from baseline & $9.7 \pm 14.7$ & $23.7 \pm 13.4$ & 0.49 & $14.0 \pm 20.0$ & $(-26.9,54.9)$ \\
\hline \multicolumn{6}{|l|}{ Disposition index } \\
\hline Baseline & $1,214 \pm 691$ & $1,721 \pm 2,014$ & 0.36 & & \\
\hline Change from baseline & $-218 \pm 158^{*}$ & $352 \pm 143^{\dagger}$ & 0.013 & $570 \pm 214$ & $(131,1,009)$ \\
\hline$\%$ Change from baseline & $-19.7 \pm 13.6^{*}$ & $45.4 \pm 12.3^{\dagger}$ & 0.002 & $65.1 \pm 18.5$ & $(27.2,103)$ \\
\hline
\end{tabular}

Baseline values are expressed as mean $\pm \mathrm{SD}$. Changes from baseline are expressed as mean $\pm \mathrm{SEM}$ (ANCOVA)

${ }^{a}$ Analyses were carried out for 17 participants with imputed values for the missing $(n=4)$ subjects using last observation carried forward

${ }^{*} p<0.05$ within group (baseline vs 13 weeks)

${ }^{\dagger} p=0.05$ within group (baseline vs 13 weeks)

$\mathrm{S}_{\mathrm{I}}$, insulin sensitivity; $\mathrm{S}_{\mathrm{G}}$, glucose effectiveness

\begin{tabular}{|c|c|c|c|c|c|}
\hline Variable & $\begin{array}{l}\text { Placebo } \\
(n=15)\end{array}$ & $\begin{array}{l}\text { HCQ } \\
(n=17)\end{array}$ & $\begin{array}{l}p \text { value } \\
\text { (HCQ vs } \\
\text { placebo) }\end{array}$ & $\begin{array}{l}\text { Difference } \\
\text { (HCQ vs } \\
\text { placebo) }\end{array}$ & $95 \% \mathrm{CI}$ \\
\hline \multicolumn{6}{|l|}{ Fasting glucose $(\mathrm{mmol} / \mathrm{l})$} \\
\hline Baseline & $5.16 \pm 0.49$ & $5.47 \pm 0.79$ & 0.20 & & \\
\hline Change from baseline & $+0.07 \pm 0.09$ & $-0.22 \pm 0.10$ & 0.04 & $-0.30 \pm 0.14$ & $(-0.58,-0.02)$ \\
\hline \multicolumn{6}{|l|}{ Fasting $\mathrm{HbA}_{1 \mathrm{c}}(\%)$} \\
\hline Baseline & $5.65 \pm 0.41$ & $5.72 \pm 0.35$ & 0.60 & & \\
\hline Change from baseline & $+0.14 \pm 0.04$ & $+0.03 \pm 0.03$ & 0.03 & $-0.11 \pm 0.05$ & $(-0.21,-0.03)$ \\
\hline \multicolumn{6}{|l|}{ Fasting insulin (pmol/l) } \\
\hline Baseline & $61.1 \pm 61.0$ & $66.7 \pm 37.5$ & 0.77 & & \\
\hline Change from baseline & $+7.0 \pm 7.0$ & $+16.0 \pm 7.0$ & 0.34 & $9.0 \pm 9.7$ & $(-9.8,28.5)$ \\
\hline \multicolumn{6}{|l|}{ Fasting NEFA $(\mu \mathrm{mol} / \mathrm{l})$} \\
\hline Baseline & $509 \pm 150$ & $538 \pm 132$ & 0.59 & & \\
\hline Change from baseline & $-31 \pm 32$ & $-74 \pm 35$ & 0.38 & $-43 \pm 48$ & $(-141,5.5)$ \\
\hline \multicolumn{6}{|l|}{ Fasting C-peptide (nmol/l) } \\
\hline Baseline & $1.32 \pm 0.67$ & $1.27 \pm 0.48$ & 0.82 & & \\
\hline Change from baseline & $+0.01 \pm 0.08$ & $+0.18 \pm 0.09$ & 0.16 & $0.17 \pm 0.12$ & $(-0.08,0.42)$ \\
\hline \multicolumn{6}{|c|}{ C-peptide/insulin molar ratio (pmol/pmol) } \\
\hline Baseline & $31.4 \pm 17.1$ & $29.2 \pm 20.3$ & 0.76 & & \\
\hline Change from baseline & $-5.5 \pm 3.3$ & $-4.8 \pm 3.6$ & 0.88 & $0.8 \pm 4.9$ & $(-9.4,10.9)$ \\
\hline
\end{tabular}

Baseline values are expressed as means $\pm \mathrm{SD}$. Changes from baseline are expressed as means $\pm \mathrm{SE}$ adjusted for baseline (ANCOVA) 
Fig. 2 (a, b) Plasma glucose concentrations in placebo (a) and HCQ (b) groups during IVGTTs. (c, d) Plasma insulin concentrations in placebo (c) and HCQ (d) groups. Black circles, baseline; white circles, after 13 weeks of treatment. Individual points are mean \pm SEM a

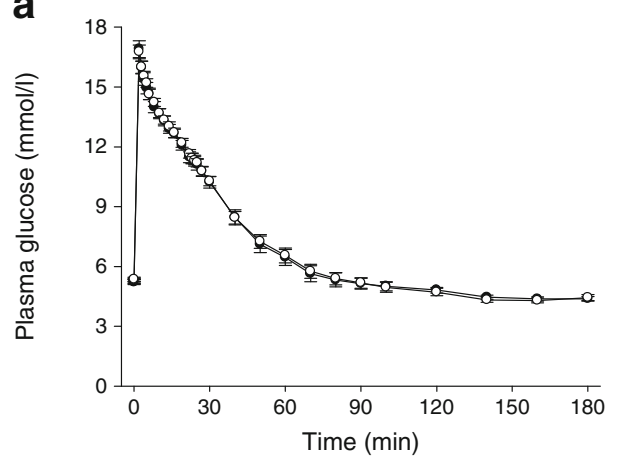

C

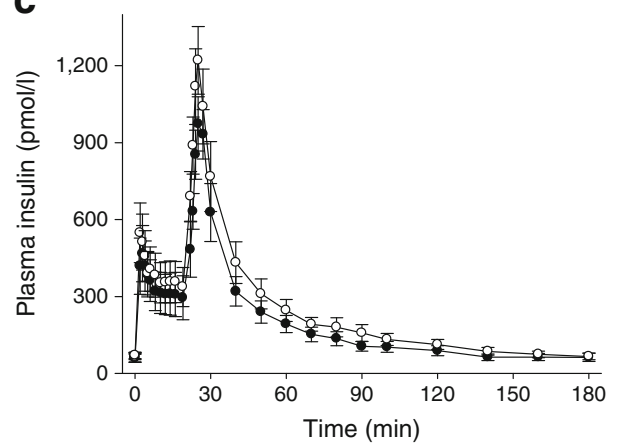

b

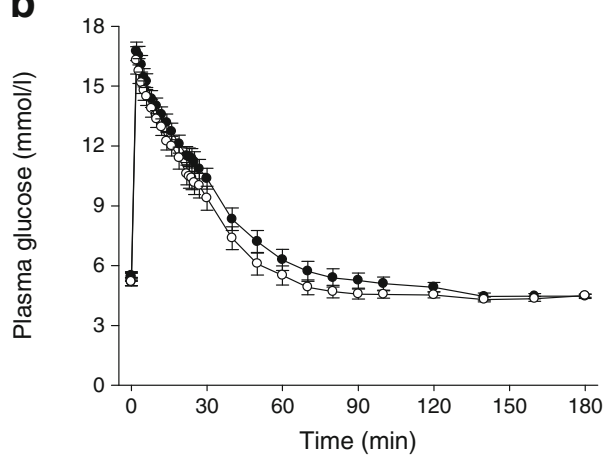

d

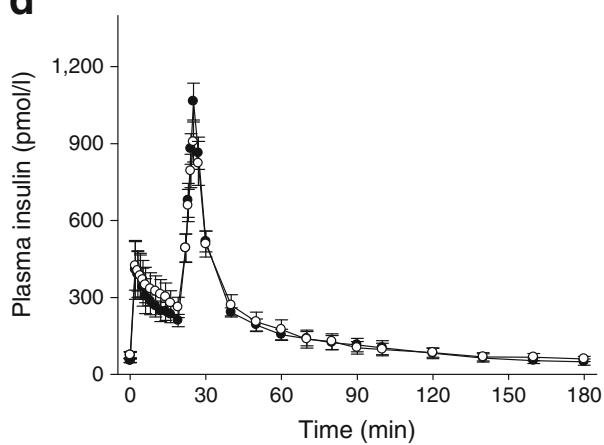

mechanisms have not been elucidated. Here, we present the results of a placebo-controlled, double-blinded study specifically designed to examine how HCQ affects glucose homeostasis. In non-diabetic individuals, HCQ treatment improved both insulin sensitivity and beta cell function. This dual effect is noteworthy since both insulin resistance and beta cell dysfunction are implicated in the progression to type 2 diabetes. HCQ also had an effect on fasting glucose concentration, indicating that the combined changes in insulin sensitivity and beta cell function were biologically relevant.

The exact effect size on insulin sensitivity was difficult to determine because the placebo group experienced an unexplained decline. However, if one considers only the change within the HCQ group $(20 \% \pm 7.1 \%)$, the magnitude is still clinically significant. For comparison, metformin has been shown to improve hepatic insulin resistance in type 2 diabetes, measured by endogenous glucose production rate, by as much as $15 \%$ [17] and $26 \%$ [18].

Recently, two small studies examined the effect of short courses of HCQ on indirect markers of insulin sensitivity but found mixed results. An open-label, crossover study of non-diabetic healthy individuals found that the Matsuda index (a surrogate of insulin sensitivity) improved after 6 weeks of HCQ treatment [19]. In contrast, another study with rheumatoid arthritis patients, also employing a crossover design, reported no improvement in the Matsuda index [20]. However, HCQ's long half-life of 44-50 days [21] can be problematic in crossover studies of short duration because of the high potential for carryover effects

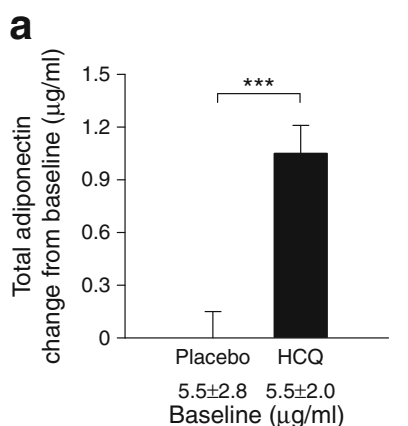

Fig. 3 Effect of HCQ on plasma adiponectin. After 13 weeks of treatment, HCQ increased the circulating concentrations of total adiponectin (a), HMW adiponectin (b) and LMW adiponectin (c). Changes are
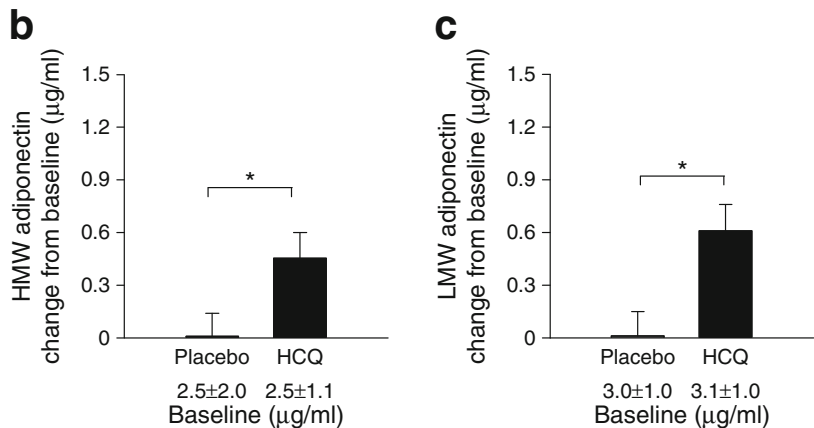

mean \pm SEM adjusted for baseline (ANCOVA). Baseline values before treatment are expressed as mean $\pm \mathrm{SD}$ and were not statistically different between groups. ${ }^{*} p<0.05$ and ${ }^{* * *} p<0.001$ between groups 
during the washout phase. Also, the Matsuda index is a surrogate measurement of insulin sensitivity and therefore can miss metabolic changes only observed by more robust methodology.

Drug-induced hypoglycaemia has been reported with chloroquine and its molecular similarity to HCQ served as a rationale for two older randomised trials to test HCQ in type 2 diabetes $[11,12]$. Both trials concluded that HCQ improves hyperglycaemia, but the physiological mechanisms have not been established. In one of those studies, insulin sensitivity was measured by IVGTT in a small subset of participants ( $n=9 \mathrm{HCQ}, n=15$ placebo), but no improvements were observed [11]. However, the study did not employ a uniform IVGTT protocol, mixing different protocols with different dextrose and insulin bolus doses, thus precluding reliable conclusions about HCQ's mechanism of action. Data from experimental models [22] and a case report [8] suggest that chloroquine, but not HCQ, may decrease insulin clearance. However, our data suggests that HCQ does not share this property with chloroquine since estimated insulin clearance was not altered by HCQ treatment.

The mechanism of action of HCQ at the molecular level is not well understood, but HCQ is employed in rheumatic diseases because it inhibits cytokine release [23]. Insulin resistance has been linked to subclinical inflammation and our initial hypothesis was that HCQ might change insulin sensitivity by ameliorating inflammatory activity. However, we found that circulating markers of inflammation were not affected by HCQ. On the other hand, those markers may be relatively insensitive for detecting tissue-level inflammation. Therefore, the possibility that HCQ's mechanism of action might involve inhibition of inflammation cannot be entirely excluded on the basis of those findings alone.

Adipose tissue inflammation has been linked to insulin resistance and lower production of adiponectin. We found that HCQ significantly increased levels of both HMW and LMW adiponectin, suggesting the possibility of antiinflammatory effects in adipose tissue. The observation that HCQ increases adiponectin concentrations is a novel finding that, to our knowledge, has not been previously described. Adiponectin has been postulated to have an insulin-sensitising effect [24] and, therefore, a potential mechanism by which HCQ might affect insulin sensitivity would be modulation of adipose tissue inflammation and adiponectin production. Less is known about the effect of adiponectin on insulin secretion but adiponectin receptors are found in beta cells [25] and upon activation enhance glucose-stimulated insulin secretion [26, 27]. It is unclear whether these actions are physiologically relevant. Further research is thus warranted to establish how HCQ improves insulin sensitivity and beta cell function.
Strengths of our study include the rigorous method used to measure insulin sensitivity and a randomised, double-blinded, placebo-controlled design. Another strength is that the improvement was observed with a dose of HCQ (400 mg/day) that is typically considered safe for long-term clinical use and is often employed in rheumatic diseases. This study also has limitations. First, there were missing data due to dropouts. However, when sensitivity analyses were employed to test different assumptions, metabolic improvements remained statistically significant. Second, insulin sensitivity measured by the minimal model combines hepatic and peripheral sensitivity. Studies using hyperinsulinaemic-euglycaemic clamps would be important to confirm the findings observed here and separate the relative contribution of hepatic vs peripheral components. Finally, because non-diabetic individuals were studied, the effects in type 2 diabetes must be inferred. Nevertheless, our design provides reassurance that the results were not an indirect consequence of reduced glucotoxicity, a potential confounder in studies involving hyperglycaemic individuals.

Since HCQ has been employed in the treatment of rheumatic conditions, our findings are relevant to patients taking this medication. For instance, changes in insulin sensitivity and beta cell function should theoretically lead to a lower risk of type 2 diabetes. In those with established diabetes, initiation of HCQ treatment should be accompanied by the awareness that improved glucose metabolism and decreased requirement for glucose-lowering drugs may occur. A notable characteristic of HCQ is that its tolerability and safety profile are well established. HCQ is generally well tolerated, inexpensive and broadly available and therefore worthy of investigation in diabetes prevention and treatment. Trials specifically designed for these purposes would be required, and our findings are supportive of such efforts.

In summary, our findings demonstrate that HCQ improves insulin sensitivity, beta cell function and adiponectin levels in non-diabetic individuals. These effects likely explain the improved glucose homeostasis that has been reported with HCQ treatment and suggest that HCQ should be further studied in disorders of glucose metabolism.

Funding This work was supported by National Institutes of Health grants no. 5R21DK082878 (FGST), UL1-RR024153 and UL1TR000005 (University of Pittsburgh).

Duality of interest The authors declare that there is no duality of interest associated with this manuscript.

Contribution statement MCMW and FGST designed the study, performed data analyses and wrote the manuscript. FGST designed and analysed the metabolism studies. TO contributed to analysis and interpretation of data. KH contributed to the acquisition of data. CKM and SFK contributed to data analysis. All authors contributed to drafting or revising the manuscript for important intellectual content and approved the final version for publication. FGST is the guarantor of this work. 


\section{References}

1. Knowler WC, Barrett-Connor E, Fowler SE et al (2002) Reduction in the incidence of type 2 diabetes with lifestyle intervention or metformin. N Engl J Med 346:393-403

2. Tuomilehto J, Lindstrom J, Eriksson JG et al (2001) Prevention of type 2 diabetes mellitus by changes in lifestyle among subjects with impaired glucose tolerance. N Engl J Med 344:1343-1350

3. DeFronzo RA, Tripathy D, Schwenke DC et al (2011) Pioglitazone for diabetes prevention in impaired glucose tolerance. N Engl J Med 364:1104-1115

4. Investigators DT, Gerstein HC, Yusuf S et al (2006) Effect of rosiglitazone on the frequency of diabetes in patients with impaired glucose tolerance or impaired fasting glucose: a randomised controlled trial. Lancet 368:1096-1105

5. Wasko MC, Hubert HB, Lingala VB et al (2007) Hydroxychloroquine and risk of diabetes in patients with rheumatoid arthritis. JAMA 298:187-193

6. Bili A, Sartorius JA, Kirchner HL et al (2011) Hydroxychloroquine use and decreased risk of diabetes in rheumatoid arthritis patients. $\mathrm{J}$ Clin Rheumatol 17:115-120

7. Solomon DH, Massarotti E, Garg R, Liu J, Canning C, Schneeweiss S (2011) Association between disease-modifying antirheumatic drugs and diabetes risk in patients with rheumatoid arthritis and psoriasis. JAMA 305:2525-2531

8. Blazar BR, Whitley CB, Kitabchi AE et al (1984) In vivo chloroquine-induced inhibition of insulin degradation in a diabetic patient with severe insulin resistance. Diabetes 33:1133-1137

9. Rees RG, Smith MJ (1987) Effect of chloroquine on insulin and glucose-homeostasis in normal subjects and patients with noninsulin-dependent diabetes-mellitus. BMJ 294:900-901

10. Smith GD, Amos TA, Mahler R, Peters TJ (1987) Effect of chloroquine on insulin and glucose homoeostasis in normal subjects and patients with non-insulin-dependent diabetes mellitus. BMJ 294: 465-467

11. Gerstein HC, Thorpe KE, Taylor DW, Haynes RB (2002) The effectiveness of hydroxychloroquine in patients with type 2 diabetes mellitus who are refractory to sulfonylureas - a randomized trial. Diabetes Res Clin Pract 55:209-219

12. Quatraro A, Consoli G, Magno M et al (1990) Hydroxychloroquine in decompensated, treatment-refractory noninsulin-dependent diabetes mellitus. A new job for an old drug? Ann Intern Med 112: 678-681

13. Monzillo LU, Hamdy O, Horton ES et al (2003) Effect of lifestyle modification on adipokine levels in obese subjects with insulin resistance. Obes Res 11:1048-1054
14. Bergman RN, Ider YZ, Bowden CR, Cobelli C (1979) Quantitative estimation of insulin sensitivity. Am J Physiol 236:E667-E677

15. Welch S, Gebhart SS, Bergman RN, Phillips LS (1990) Minimal model analysis of intravenous glucose tolerance test-derived insulin sensitivity in diabetic subjects. J Clin Endocrinol Metab 71:15081518

16. Bojsen-Moller KN, Dirksen C, Jorgensen NB et al (2013) Increased hepatic insulin clearance after Roux-en-Y gastric bypass. J Clin Endocrinol Metab 98:E1066-E1071

17. Cusi K, Consoli A, DeFronzo RA (1996) Metabolic effects of metformin on glucose and lactate metabolism in noninsulin-dependent diabetes mellitus. J Clin Endocrinol Metab 81:4059-4067

18. DeFronzo RA, Barzilai N, Simonson DC (1991) Mechanism of metformin action in obese and lean noninsulin-dependent diabetic subjects. J Clin Endocrinol Metab 73:1294-1301

19. Mercer E, Rekedal L, Garg R, Lu B, Massarotti EM, Solomon DH (2012) Hydroxychloroquine improves insulin sensitivity in obese non-diabetic individuals. Arthritis Res Ther 14:R135

20. Solomon DH, Garg R, Lu B et al (2014) Effect of hydroxychloroquine on insulin sensitivity and lipid parameters in rheumatoid arthritis patients without diabetes mellitus: a randomized, blinded crossover trial. Arthritis Care Res 66:1246-1251

21. Tett SE, Cutler DJ, Day RO, Brown KF (1989) Bioavailability of hydroxychloroquine tablets in healthy volunteers. Br J Clin Pharmacol 27:771-779

22. Bevan AP, Krook A, Tikerpae J, Seabright PJ, Siddle K, Smith GD (1997) Chloroquine extends the lifetime of the activated insulin receptor complex in endosomes. J Biol Chem 272:26833-26840

23. van den Borne BE, Dijkmans BA, de Rooij HH, le Cessie S, Verweij CL (1997) Chloroquine and hydroxychloroquine equally affect tumor necrosis factor-alpha, interleukin 6, and interferongamma production by peripheral blood mononuclear cells. J Rheumatol 24:55-60

24. Ye R, Scherer PE (2013) Adiponectin, driver or passenger on the road to insulin sensitivity? Mol Metab 2:133-141

25. Kharroubi I, Rasschaert J, Eizirik DL, Cnop M (2003) Expression of adiponectin receptors in pancreatic beta cells. Biochem Biophys Res Commun 312:1118-1122

26. Rao JR, Keating DJ, Chen C, Parkington HC (2012) Adiponectin increases insulin content and cell proliferation in MIN6 cells via PPAR $\gamma$-dependent and PPAR $\gamma$-independent mechanisms. Diabetes Obes Metab 14:983-989

27. Wijesekara N, Krishnamurthy M, Bhattacharjee A, Suhail A, Sweeney G, Wheeler MB (2010) Adiponectin-induced ERK and Akt phosphorylation protects against pancreatic beta cell apoptosis and increases insulin gene expression and secretion. J Biol Chem 285:33623-33631 